\title{
Improved Shaping of Reflector Antennas using a New Minimax Initialization Strategy
}

\author{
Eltved, Anders; Andersen, Martin Skovgaard ; Borries, Oscar
}

Published in:

Proceedings of 2018 International Applied Computational Electromagnetics Society Symposium

Link to article, DOI:

10.23919/ROPACES.2018.8364326

Publication date:

2018

Document Version

Peer reviewed version

Link back to DTU Orbit

Citation $(A P A)$ :

Eltved, A., Andersen, M. S., \& Borries, O. (2018). Improved Shaping of Reflector Antennas using a New Minimax Initialization Strategy. In Proceedings of 2018 International Applied Computational Electromagnetics Society Symposium IEEE. https://doi.org/10.23919/ROPACES.2018.8364326

\section{General rights}

Copyright and moral rights for the publications made accessible in the public portal are retained by the authors and/or other copyright owners and it is a condition of accessing publications that users recognise and abide by the legal requirements associated with these rights.

- Users may download and print one copy of any publication from the public portal for the purpose of private study or research.

- You may not further distribute the material or use it for any profit-making activity or commercial gain

- You may freely distribute the URL identifying the publication in the public portal 


\section{Improved Shaping of Reflector Antennas using a New Minimax Initialization Strategy}

\author{
Anders Eltved* and Martin S. Andersen \\ DTU Compute, Technical University of Denmark \\ Kongens Lyngby, Denmark \\ *Email: aelt@dtu.dk
}

\author{
Oscar Borries \\ TICRA \\ Copenhagen, Denmark
}

\begin{abstract}
Numerical optimization is an essential part of the design process when shaping a contoured beam reflector antenna to produce a required coverage. The minimax formulation that has been used for decades generally results in a nonconvex problem, and since the evaluation of a design is costly, local optimization is typically used in practice. An inherent challenge with the minimax formulation is that it often leads to multiple local minima. As a result, the quality of the final design obtained via local optimization depends strongly on the initial design.

To address the sensitivity to the initial design, we investigate the use of initialization based on a different problem formulation. In particular, we first solve a one-sided nonlinear least-squares problem to improve an initial design, and the resulting design is then used as initialization of a minimax algorithm. We use this strategy to shape a reflector antenna starting from different initial shapes, and our results show that the initialization strategy improves the coverage with five out of six initial guesses.
\end{abstract}

\section{INTRODUCTION}

For many modern communications satellites, the design of contoured beam reflector antennas involves the use of optimization to shape the reflector to produce a desired coverage. The performance of a reflector system is simulated using Physical Optics (PO) which is sometimes augmented by Physical Theory of Diffraction (PTD) to account for the effects from the reflector edges. Using these techniques, the gain, cross-polar performance, and other relevant quantities can be evaluated for a specific design.

To optimize the design, it is common to consider the minimax problem where the objective is to maximize the minimum gain in the coverage, or equivalently, maximizing the guaranteed gain in the coverage. The minimax problem has been studied extensively [1], and good local optimization algorithms exist for solving the problem [2], [3]. However, with local optimization algorithms, the solution depends on an initial guess which is usually provided by the user. In practice, the antenna designer comes up with an initial design which is then improved by the optimization algorithm.

Unfortunately, the minimax formulation can have many local minima, and hence local optimization algorithms can be sensitive to the initial guess; if the antenna designer provides a poor initial design, the final design after optimization may not be improved much. Multi-start methods (doing multiple local optimizations from various starting points) and global optimization methods can improve this situation, but evaluating the performance of a design, which is done in each iteration of the optimization, can take hours in some applications, making these approaches too computationally expensive. In practice, the user often decides on a maximum number of function evaluations and the optimization is terminated if this number is exceeded even if the usual stopping criteria are not satisfied.

In this study, we present an alternative optimization problem to solve - the one-sided nonlinear least-squares (onesided LS) problem - and an algorithm for finding a (local) minimum. Given a maximum number of function evaluations, $k_{\max }$, to be used in the optimization, we propose the following strategy: (i) the initial design is first improved by optimizing the one-sided LS formulation using at most $k_{\max } / 2$ iterations (one iteration corresponds to one function evaluation), and (ii) the resulting design is then optimized using the minimax algorithm with a cap on the number of iterations such that the total number of function evaluations is at most $k_{\max }$.

\section{METHOD}

In the following, we will assume that $x \in \mathbb{R}^{n}$ is a vector of $n$ parameters that define the design of the reflector antenna (e.g., the elements of $x$ can represent spline coefficients of a shaped surface, the position or excitation of feeds and arrays, etc.). For each of the $m$ points in the coverage we define a residual function:

$$
r_{i}(x)=w_{i}\left(g_{i}-f_{i}(x)\right),
$$

where $f_{i}(x)$ is a function that measures the performance of the current design, $g_{i}$ is a performance goal, and $w_{i}$ is a weight that determines the importance of meeting the performance goal at the $i$ th point. To ensure that a design can be realized, it is often necessary to put constraints on $x$ (e.g., restricting the curvature of the reflector to avoid having to bend the metal surface of the reflector too much, or restricting the position of a feed such that it does not coincide with other components on the structure). Here we consider linear constraints represented by the set $\mathcal{C}=\left\{x \in \mathbb{R}^{n} \mid C x+d \geq 0\right\}$, where $C \in \mathbb{R}^{p \times n}$, $d \in \mathbb{R}^{p}, p$ is the number of constraints, and the inequality is element-wise. The minimax problem can now be formulated as:

$$
\underset{x \in \mathcal{C}}{\operatorname{minimize}} \max _{i} r_{i}(x)
$$


and the one-sided LS problem is given by:

$$
\underset{x \in \mathcal{C}}{\operatorname{minimize}} \sum_{i=1}^{m} \max \left\{0, r_{i}(x)\right\}^{2},
$$

Notice that all positive residuals are penalized in the onesided LS formulation; this is sensible since a positive residual implies that the goal is not attained at the given point. As mentioned in the introduction, there are efficient algorithms for optimizing (2) locally, and in the next section, we outline a method for local optimization of (3).

\section{Algorithm}

To optimize (3), we consider the equivalent problem:

$$
\begin{array}{ll}
\underset{x \in \mathcal{C}, u \in \mathbb{R}^{m}}{\operatorname{minimize}} & \|u\|^{2} \\
\text { subject to } & u_{i} \geq r_{i}(x), u_{i} \geq 0, \quad i=1, \ldots, m,
\end{array}
$$

where $u$ is a vector of auxiliary variables. This problem has a quadratic objective function, but due to the possibly nonconvex constraints $u_{i} \geq r_{i}(x)$, it is still intractable to find a global minimum. Thus, we use a trust-region method [4] to search for a local minimum. At a current iterate, $x^{k}$, we linearize the residual functions in (4), and find a candidate for the next iterate, $x^{k+1}=x^{k}+\Delta x$, by solving the trust-region subproblem:

$$
\begin{aligned}
\underset{\Delta x \in \mathbb{R}^{n}, u \in \mathbb{R}^{m}}{\operatorname{minimize}} & \|u\|^{2} \\
\text { subject to } & C \Delta x+d^{k} \geq 0 \\
& u \geq r^{k}+J^{k} \Delta x \\
& u \geq 0, \delta^{k} \geq\|\Delta x\|_{\infty},
\end{aligned}
$$

where $\delta^{k}>0$ is the trust-region radius, $r^{k}=r\left(x^{k}\right)$, and:

$$
d^{k}=C x^{k}+d, J^{k}=\left[\nabla r_{1}\left(x^{k}\right) \cdots \nabla r_{m}\left(x^{k}\right)\right]^{T} .
$$

The problem (5) is a convex quadratic problem with linear constraints (since the $\infty$-norm is linearly representable) which we solve with an interior-point method [5].

\section{Results}

To illustrate the effect of the initialization strategy, we consider a case where the objective is to shape a reflector for illumination of the continental United States (the US excluding Alaska and Hawaii). We have $n=783$ variables (spline coefficients of a reflector surface) and optimize the coverage over $m=810$ points starting from six different initial shapes. The initial shapes were created by de-focusing the reflector such that the initial guesses become progressively worse: guess 1 is expected to produce the best results and initial guess 6 is expected to produce the worst results. We did not impose constraints on $x$ (i.e., $p=0$ ). An example of the coverage obtained with the minimax algorithm initialized with initial guess 2 is shown in Fig. 1.

In our experiments, we limited the number of function evaluations $\left(k_{\max }\right)$ to 500 , and for each of the six initial guesses we did two experiments. In the first experiment, we

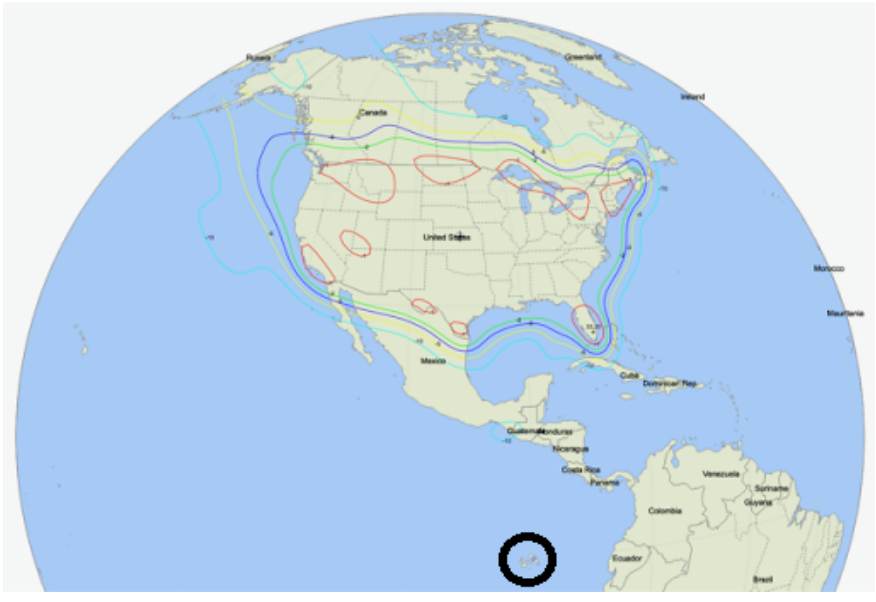

Fig. 1. Contours of coverage optimized using the minimax algorithm. The peak gain of around $33 \mathrm{~dB}$ is at the cross in Florida. The Nadir direction of the satellite is shown in the black circle.

TABLE I. VAlue of MaXimal Residual in DB AfTer 500 Iterations. The 6 Initial Guesses Become Progressively Worse: 1 IS A Good (Green) Initial Guess and 6 is a Poor (Red) Initial Guess.

\begin{tabular}{|l|r|r|r|r|r|r|}
\hline Initial Guess & 1 & 2 & 3 & 4 & 5 & 6 \\
\hline Minimax only & -0.074 & 0.090 & 0.826 & 2.019 & 4.149 & 9.360 \\
OLS+minimax & -0.074 & -0.004 & 0.417 & 1.952 & 4.103 & 7.690 \\
\hline Difference & 0.000 & 0.094 & 0.409 & 0.067 & 0.046 & 1.67 \\
\hline
\end{tabular}

applied the one-sided LS algorithm for 250 iterations (or until a stopping criteria is met), followed by the minimax algorithm for the remaining iterations. In the second experiment, we used only the minimax algorithm for 500 iterations. Our results are summarized in Table I; recall that smaller residuals are better. The results clearly show that the initialization strategy can improve the minimum gain in the coverage: the gain is improved for initial guesses 2-6, and the result remains the same for initial guess 1. Considering the maximal residualit measures how far we are from reaching the goal value - the absolute improvement is largest for initial guess $6(1.67 \mathrm{~dB})$, where there is much room for improvement, and the relative improvement is largest for initial guess 3 (49.5\%).

\section{CONClusion}

The use of one-sided least-squares for initializing the minimax algorithm can lead to improved results: in our numerical experiments, the initialization strategy led to an improved design with five out of six initial guesses.

\section{REFERENCES}

[1] V. Demyanov and V. Molozemov, Introduction to Minimax. Wiley: New York, 1974

[2] J. Hald, "Two stage algorithms for linearly constrained minimax optimization," Ph.D. dissertation, Technical University of Denmark, 1984.

[3] K. Madsen and H. Schjær-Jacobsen, "Linearly constrained minimax optimization," Mathematical Programming, vol. 14, no. 1, 1978.

[4] A. Conn, N. Gould, and P. Toint, Trust Region Methods. Society for Industrial and Applied Mathematics, 2000.

[5] J. Nocedal and S. Wright, "Quadratic programming," in Numerical Optimization, 2nd ed. Springer-Verlag New York, 2006, ch. 16. 